\title{
Introducing First Aid Skills to Pre-Clerkship Medical Students While Reinforcing Basic Science Concepts
}

\author{
Brian B. Wexler • Norma S. Saks
}

Published online: 13 March 2014

(C) International Association of Medical Science Educators 2014

The benefit of exposing medical students throughout their medical training to emergency medicine concepts, including basic first aid knowledge, has been established [1]. The American College of Emergency Physicians (2008) called for introduction and integration of basic first aid skills throughout the 4 years of medical school [2]. Teaching clinical skills to preclinical students has the benefit of enhancing learning by providing meaningful clinical relevance to basic science learning, is motivating for learning, and is beneficial for reviewing and reinforcing basic science concepts [3].

It is often assumed that entering medical students have knowledge and skills to provide essential medical care; however, many are unfamiliar with even very basic first aid skills. A 2-hour workshop to teach first aid procedures was developed and presented to 25 volunteer first (M1) and second year (M2) students at Rutgers Robert Wood Johnson Medical School (RWJMS). The workshop covered procedures for wound care, hemorrhage control, and treatment of burns. Relevant basic science content (musculoskeletal anatomy and physiology, microbiology of tetanus and skin infections, and antibiotic pharmacology) was presented, followed by demonstrations and hands-on practice sessions. The practice sessions included procedures for applying direct pressure to a wound, dressing a wound, and wrapping burns with sterile gauze.

\footnotetext{
B. B. Wexler

Rutgers Robert Wood Johnson Medical School, Piscataway, NJ 08854, USA

N. S. Saks $(\bowtie)$

Department of Psychiatry, Rutgers Robert Wood Johnson Medical

School, 675 Hoes Lane, V-01, Piscataway, NJ 08854, USA

e-mail: norma.saks@rwjms.rutgers.edu
}

Workshop evaluations via pre and post-surveys measured knowledge acquisition, comfort in providing first aid, and overall effectiveness of the session. Evaluation results were very positive. The mean score (12 questions) of first aid knowledge increased from $38.3 \%$ to $81.7 \%$ $(p<0.001)$. On a Likert scale measuring student comfort in providing care to a bleeding patient $(1=$ very uncomfortable to $5=$ very comfortable), scores increased from 2.68 to $4.36(p<0.001)$; comfort for providing burn care increased from 2.28 to $4.48(p<0.001)$. On additional posttest Likert scale questions $(1=$ strongly disagree to $5=$ strongly agree), students also responded very positively to the integration of basic science content (4.72), hands-on practice sessions (4.58), usefulness of information (4.76), and interest level and quality of presentation (4.76). There were no significant differences between M1 and M2 students; at least one semester of medical school basic science material is recommended. Based on this initial success, another workshop about limb injuries and cervical spine injury care is being developed as another optional student experience, and will include both basic science correlations and hands-on practice sessions.

\section{References}

1. McLaughlin SA, Hobgood C, Binder L, Manthey DE (2005) Impact of the liaison committee on medical education requirements for emergency medicine education at US schools of medicine. Acad Emerg Med 12(10):1003-1009

2. American College of Emergency Physicians (2008) Guidelines for undergraduate education in emergency medicine, viewed 7th April 2012, http://www.acep.org/content.aspx?id=29866

3. Wilson AB, Ross C, Petty M, Williams JM, Thorp LE (2009) Bridging the transfer gap: laboratory exercise combines clinical exposure and anatomy review. Med Educ 43(8):790-798 